\title{
Causal and mediating factors for anxiety, depression and well-being
}

Peter Kinderman, Sara Tai, Eleanor Pontin, Matthias Schwannauer, Ian Jarman and Paulo Lisboa

\section{Background}

The relationship between well-being and mental ill health is complex; people may experience very low levels of well-being even in the absence of overt mental health problems.

\section{Aims}

This study tested the hypothesis that anxiety, depression and well-being have different causal determinants and psychological mediating mechanisms.

\section{Method}

The influence of causal and mediating factors on anxiety, depression and well-being were investigated in a crosssectional online questionnaire survey hosted on a UK national broadcasting website.

\section{Results}

Multivariate conditional independence analysis of data from
27397 participants revealed different association pathways for the two constructs. Anxiety and depression were associated with negative life events mediated by rumination; low levels of subjective well-being were associated with material deprivation and social isolation, mediated by adaptive coping style.

\section{Conclusions}

Our findings support the 'two continua' model of the relationship between psychological well-being and mental health problems, with implications for both treatment and prevention.

\section{Declaration of interest}

None.

\section{Copyright and usage}

(c) The Royal College of Psychiatrists 2015.
The relationships between mental health, well-being and mental illness are complex, and people may experience very low levels of well-being even in the absence of overt mental health problems. ${ }^{1}$ Despite general agreement that these three concepts are important targets for health and social care, there is no consensus about the relationship between them. This conceptual distinction is best exemplified in the 'two continua' model, ${ }^{2}$ which suggests that mental illness and mental well-being are related yet distinct phenomena, rather than ends of a single measurement continuum. One important way to disambiguate 'psychological well-being', 'mental health' and 'mental health problems' is to explore their distinct causal determinants. We report here on an analysis of a unique database of 27397 participants, using the novel but powerful techniques of conditional independence graphical analysis, ${ }^{3,4}$ testing whether the same patterns of these causal factors and psychological mediators are implicated in the development of mental health problems and mental well-being. Although conditional independence mapping has been used to analyse a range of data in both social and physical sciences, this is the first time that the technique has been used on multivariate data such as these in such a substantial data-set. Conditional independence mapping has similarities with covariance or structural equation modelling, but makes fewer a priori assumptions about the expected relationships, allowing all possible permutations of association to be tested simultaneously in a single multivariate model. Identified significant associations can then be explored in more detail to explore the contingent relationships between selected variables.

\section{Method}

Participants were self-selected respondents to an online battery of questionnaires hosted on the British Broadcasting Corporation (BBC) website (the Stress Test). In total 32827 respondents (age 18-85 years) completed the test battery; their mean age was 40.5 years (s.d.=14.3), 61\% were women, 93\% were 'White British' or 'White other' and $88 \%$ were from the UK. To determine whether the sample was representative of the UK population, results were compared with national data for England and Wales. ${ }^{5}$

\section{Procedure}

The Stress Test was promoted via multimedia formats (television, radio and online) and launched on BBC Radio 4's All in the Mind, a flagship documentary focusing on issues of the human mind. The test's URL (www.bbc.co.uk/labuk/experiments/stress/) was publicised on subsequent episodes, related television broadcasts and made available on BBC web pages and social networking sites. Visitors to the Stress Test's homepage accessed the test by signing in using BBC online membership. The test was 'live' for a 4-week period. The test had 12 sections, which took approximately $20 \mathrm{~min}$ to complete in total. It was designed by authors P.K. and S.T. and developed in collaboration with BBC Lab UK. Questionnaire items were completed in a fixed order and answers were selected from a drop-down menu. Some tasks were constrained within time limits. On completion, an overview of scores was displayed on a results home page and URL links for comprehensive and tailored feedback based on test scores were presented. Once participants had completed the test they were not permitted to retake it.

\section{Measures}

Measures were selected on the basis of theoretical principles and empirical research to provide indicators of latent constructs representative of the components of the biopsychosocial model of mental health and well-being. ${ }^{6}$ Where measures were modified or created for the purposes of this study, other analyses have demonstrated their post hoc construct validity. ${ }^{7}$ Demographic data collected included age, gender, ethnic group, occupation, gross annual or weekly household earnings, highest level of formal schooling, occupational status, parents' income, relationship 
status and number of children. Measured variables to represent the biological component of the theoretical model were a yes/no response to indicate participants' reports of familial mental health diagnoses by a psychiatrist or general practitioner, ${ }^{8}$ and performance on two cognitive tests to detect response to negative feedback and negative and positive stimuli. These were the 'delayed match to sample' (DMS) and the 'affective go/no go' (AGN) tasks adapted from the Cambridge Neuropsychological Test Automated Battery (CANTAB). ${ }^{9-11}$ These tests were chosen to measure neurological functioning previously associated with anxiety and depression, ${ }^{9,10}$ and therefore supplement the more subjective participant report of familial history of mental health problems. The social component of the model was represented by a novel 11-item questionnaire designed to indicate social relationships with friends and family, and participation in social activities. ${ }^{12}$ These items were a combination of Likert scale and yes/no responses. Indicators of the circumstantial component included recent and historical life events. Recent life events were measured using the List of Threatening Experiences Questionnaire (LTE-Q). ${ }^{13}$ Participants also indicated on a five-point Likert scale whether they believed they had historically been physically, sexually or emotionally abused, or bullied at school. ${ }^{14}$ The two key psychological processes (response style and attributional style) were measured using an adapted Response Style Questionnaire (RSQ). ${ }^{15}$ In the modified version for the Stress Test, participants indicated on a Likert scale their response to stressful situations from a list of coping strategies pertaining to rumination, problem-solving/adaptive or dangerous activities. Rumination was characterised by endorsement of statements such as 'I think about my shortcomings, failings, faults and mistakes'; adaptive coping was characterised by endorsement of statements such as 'I make a plan to overcome a problem'; and dangerous activities encompassed strategies such as 'go shopping with no regard for the debts I may run up' or 'drink alcohol excessively'. Attributional style was measured using an adapted and modified version of the Internal, Personal and Situational Attributions Questionnaire (IPSAQ), ${ }^{16}$ to determine the degree to which individuals generate internal, personal or situational causes for an event or situation. Finally, mental health problems were assessed using the wellvalidated Goldberg Anxiety and Depression (GAD) scales, ${ }^{17}$ a paired nine-item self-report symptom inventory designed to assess anxiety and depression. The GAD scale was chosen for its brevity and because it has been found to be a valid and acceptable method of detecting psychological disturbance in the general population. ${ }^{17}$ Well-being was measured using the BBC Subjective Well-Being (BBC-SWB) scale, ${ }^{18}$ a 24 -item questionnaire designed to measure people's subjective experiences across the wide breadth of domains commonly included in the definitions of well-being: psychological well-being, physical health and satisfaction with relationships. The BBC-SWB scale has been found to be a reliable and valid measure for the online assessment of well-being in the general population with good psychometric properties. ${ }^{19}$

\section{Statistical analysis}

The data-set was subjected to conditional independence graphical analysis, ${ }^{3,4}$ to test the conditional independence between paired variables. Since mutual covariance is common in multivariate analyses - and especially common in the social sciences - the algorithm used in this analysis ensures that each specific test for significance of association is conditional on all associations with other variables connected in the model. The resulting multivariate association structure is represented in a conditional independence map. Any pair of variables that do not carry significant information about each other are disconnected in the map. The remaining associations are then directed according to the relative strength of conditional probabilities, and subjected also to additional constraints so that the final map is consistent and acyclic, so removing any closed loops. Specific associations between variables may be investigated further using covariance tables. Missing data were deleted listwise to produce complete data for 27397 participants (retention of $83.5 \%$ of the original sample, $n=32827$ ). The neurocognitive data accounted for a large proportion of missing data owing to invalid recording, probably because of technical error. Missing case analysis revealed no significant difference between those with and without missing data on demographic variables and a selection of measured variables.

The software used in this study, CiMAp version $1.0,{ }^{3}$ implemented within the MatLab (R2011a) 64-bit (win64) statistics package, employs a computationally efficient algorithm, ${ }^{28}$ with adjustments for repeated tests. It starts from a fully connected undirected map and deletes conditionally independent associations in multiple statistical tests. Conditional mutual information is computed using the equation

$$
\tilde{I}(i, j \mid A)=\sum_{x_{i}, x_{j}, x_{A}} p_{i j A}\left(x_{i}, x_{j}, x_{A}\right) \log _{2} \frac{p_{i j A}\left(x_{i}, x_{j} \mid x_{A}\right)}{p_{i \mid A}\left(x_{i} \mid x_{A}\right) p_{j \mid A}\left(x_{j} \mid x_{A}\right)}
$$

where $x_{i}, x_{j}, x_{A}$ represent values taken from variables $X_{i}, X_{j}$ and $X_{A}$, respectively, and $p$ is the joint mass function that is typically approximated with the joint estimate $n_{i j A} / N$.

Larger values of $\tilde{I}$ imply stronger dependencies, and significance tests assumed a $\chi^{2}$ distribution for independent or conditional independent variables. ${ }^{20}$ Further details of this equation and its use in conditional independence mapping can be found in more specialist publications. ${ }^{20}$

Conditional independence graphical analysis conducts simultaneous tests of association on categorical data. Variables with more than 15 categories (attribution style, dangerous activities response style, rumination response style, well-being score and GAD score) were transformed into deciles. Frequency of childhood abuse was transformed into six categories, owing to the distribution of responses. Family history of mental health problems was transformed to two simple 'yes/no' categories. Other variables (highest level of formal schooling achieved, occupational status, total gross annual or weekly household income, parents' income while growing up, relationship status, number of children, relationships with friends, relationships with family, social activities, frequency of social contacts, total number of life events, adaptive response style and dangerous activities response style) were used in their raw form, with each integer treated as a separate category. In the case of total gross annual income, $12 \%$ of participants recorded either 'don't know' or declined to answer the question. As these responses are meaningful (and were associated with occupational status as students or unemployed), these categories were included in the analysis rather than excluded as missing. A summary of the data processing applied to each variable is given as an online supplement (Table DS1).

\section{Results}

The majority of respondents held an undergraduate, professional qualification or postgraduate degree $(73 \%)$, were in full- or parttime employment $(73 \%)$, were in a relationship $(74 \%)$ and had no children (54\%). Compared with national data for England and Wales, ${ }^{5}$ more UK respondents were White, had slightly higher earnings and were better educated than the general population, although comparable on other demographic features. Demographic data for the full sample are presented in Table 1. 


\begin{tabular}{|c|c|c|}
\hline & $\begin{array}{l}\text { Whole sample } \\
\begin{array}{c}(n=32827) \\
\%(n)\end{array}\end{array}$ & $\begin{array}{c}\text { Missing data } \\
\text { removed } \\
(n=27397) \\
\%(n)\end{array}$ \\
\hline \multicolumn{3}{|l|}{ Ethnic group } \\
\hline White (British, Irish, other) & $85.6(28$ 105) & $92.8(25434)$ \\
\hline Black minority ethnic & $5.6(1850)$ & $5.9(1612)$ \\
\hline Rather not say or missing & 8.7 (2872) & $1.3(351)$ \\
\hline \multicolumn{3}{|l|}{ Highest level of schooling achieved } \\
\hline Did not complete schooling & $2.3(747)$ & $2.2(601)$ \\
\hline In education until age 18 years & $24.4(8032)$ & 24.7 (6766) \\
\hline Degree or professional qualification & $73.2(24048)$ & $73.1(20030)$ \\
\hline \multicolumn{3}{|l|}{ Occupational status } \\
\hline In education & $11.8(3885)$ & $11.4(3109)$ \\
\hline In employment & 73.1 (23991) & 73.7 (20 195) \\
\hline Other & $15.1(4951)$ & 14.9 (4093) \\
\hline \multicolumn{3}{|l|}{ Total gross annual household income } \\
\hline Up to $£ 39999$ ( $\$ 65000$ ) per annum & $51.6(16936)$ & $51.9(14206)$ \\
\hline Above f39 999 ( $\$ 65000$ ) per annum & $34.2(11225)$ & $36.0(9851)$ \\
\hline Don't know/prefer not to say & $14.2(4666)$ & $12.2(3340)$ \\
\hline \multicolumn{3}{|l|}{ Estimated parents' income while growing up } \\
\hline Lower than $50 \%$ population & $51.2(16824)$ & 50.8 (13913) \\
\hline Higher than $50 \%$ population & $48.7(16003)$ & $49.2(13484)$ \\
\hline \multicolumn{3}{|l|}{ Relationship status } \\
\hline In a relationship & $74.3(24389)$ & $73.2(20062)$ \\
\hline Single & $25.7(8438)$ & $26.8(7335)$ \\
\hline \multicolumn{3}{|l|}{ Number of children } \\
\hline None & 54.5 (17900) & $53.7(14717)$ \\
\hline One or more & 45.6 (14 927) & $46.3(12680)$ \\
\hline
\end{tabular}

The results of the analysis are summarised in the conditional independence map (Fig. 1), which shows variables that are significantly associated $(P<0.05)$, having tested for all possible associations. Each connecting line in Fig. 1 therefore represents the associated pairwise mutual information between the respective variables. Higher mutual information corresponds to a stronger pairwise association, so for this map the strongest pairwise association is 0.35 and the weakest 0.01 , but all are significant at the $95 \%$ confidence level. Inspecting the map in more detail reveals that the variable 'mental health problems' was directly and significantly associated with two other variables: 'rumination' (0.35) and 'number of negative life events' (0.05). This means that, given all the possible associations of 'mental health problems' with every other variable in the data, it was only these two variables that conveyed statistically significant conditional mutual information. In contrast, 'well-being' was directly and significantly associated with eight other variables: adaptive coping (0.1), frequency of social contacts (0.05), social activities (0.19), relationships with friends $(0.11)$, relationships with family (0.05), parental income (0.02), household income (0.03) and highest level of education (0.02). Surprisingly, well-being and mental health had no directly associated variables in common. All the variables included in the analysis (listed in online Table DS1) were represented on the map, with the exception of 'attribution style', which had no significant association with any other variable.

Examination of the map of conditional associations reveals a cluster of social variables associated with well-being, accompanied by adaptive coping, previously identified as a mediating variable. ${ }^{7}$ In contrast, a cluster of variables including negative life events, abuse and a familial history of mental health problems was associated with mental health problems (anxiety and depression), accompanied by rumination, again previously identified as a mediating variable. ${ }^{7}$ This analysis does not suggest that well-being and mental health problems are unrelated, merely that conditional mutual information concerning their association is best represented through a pattern of indirect associations as presented in Fig. 1 and involving several different pathways. The analysis does, however, strongly suggest that the two concepts are unlikely to be two poles of a single continuum. These associations were explored in greater detail using simple contingency tables based on median splits. Table 2 presents the data from the BBC-SWS and the GAD, with a clear inverse relationship between the two variables.

\section{Different pathways for mental health problems and well-being}

Contingency tables also permit more detailed exploration of three-way associations revealed by the conditional independence map. These analyses reveal clearly differentiated mediated pathways leading from causal factors to well-being and mental health problems. The contingent relationship between life events, rumination and mental health problems is presented in Table 3, with simple binomial categorisation to permit straightforward interpretation. For the whole sample the relationship between life events and mental health problems was relatively weak, with only a slightly disproportionate number of people falling into the cell representing both higher levels of mental health problems and more life events. The mediating effects of rumination are clear, however, in the two succeeding parts of Table 3. For participants reporting low levels of rumination, prevalence of mental health problems was low, even in the presence of more frequent life events (17\%). In contrast, for participants reporting high levels of rumination, more frequent life events were associated with a markedly higher rate of mental health problems (56\%).

Similar contingency tables explored the three-way associations between well-being, social activities and adaptive coping (Table 4). For the whole sample the relationship between social activities and well-being was clear, with higher levels of social activities associated with higher levels of subjective well-being. Again, however, adaptive coping acted as a mediating protective factor, as high levels of adaptive coping were associated with disproportionately fewer people reporting low levels of subjective well-being in the absence of social activity.

\section{Discussion}

Our findings provide clear quantitative support for the hypothesis that subjective well-being and mental health problems have distinct causal pathways, ${ }^{1,2}$ with different causal factors and psychological mediators, despite the high correlation between these concepts. These findings are strongly supportive of previous reports that a family history of mental health difficulties, social deprivation and traumatic or abusive life experiences are associated with higher levels of anxiety and depression, mediated by adaptive coping and rumination. ${ }^{7}$ The mediating role of psychological processes was strongly evidenced in the relationship between life events and mental health problems, in that there was a striking distinction between the rates of mental health problems following frequent life events for people with low, as opposed to high, levels of rumination. Low levels of subjective well-being were associated with social isolation and low levels of adaptive coping. On the other hand, mental health problems were associated with negative life events and rumination.

Because this study was conducted on a cross-sectional rather than a longitudinal basis, we were able to identify patterns of association rather than direct causal relationships. For instance, rumination is often considered a symptom of depression rather 


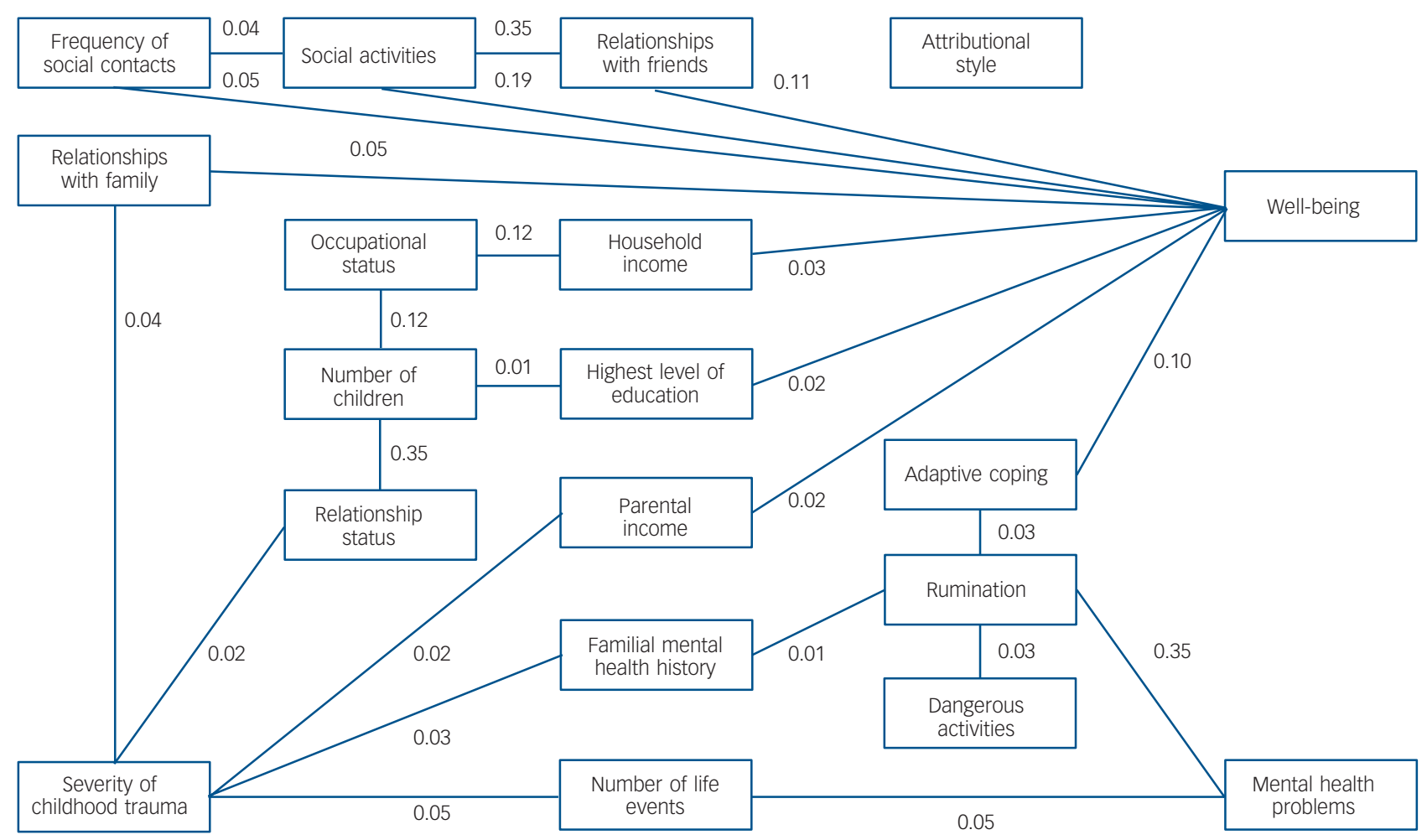

Fig. 1 Conditional independence map. Multivariate associations were calculated using mutual information, $\tilde{l}$, shown pairwise in mbits, and mapped taking account of all orders of conditioning $(P<0.05)$. This removes pairwise associations that can be explained by other variables.

\begin{tabular}{|c|c|c|}
\hline & $\begin{array}{c}\text { Low well-being } \\
n(\%)\end{array}$ & $\begin{array}{c}\text { High well-being } \\
n(\%)\end{array}$ \\
\hline \multicolumn{3}{|c|}{ All participants ( $n=27397$ ) } \\
\hline Low & $4563(16.7)$ & $10956(40.0)$ \\
\hline High & 9421 (34.4) & $2457(9.0)$ \\
\hline
\end{tabular}

than a cause or a mediator. Our findings require further confirmation in a longitudinal, predictive, design. Nevertheless, the conditional independence maps identified pathways that are consistent with current understanding of causal mechanisms. ${ }^{7,21}$ The external validity of the findings may also be subject to selfselection biases given the online nature of the survey. Responses to questions about mental distress and well-being may be affected by gender, education, socioeconomic status or a personal history of mental health problems. The UK sample in this study was less ethnically diverse, had slightly higher earnings and was better educated than the general population in the UK, although other demographic features were comparable.

\section{The two-continua model}

These findings are theoretically and clinically significant, and validate the relatively novel analytical technique of conditional independence graphical analysis. ${ }^{3,4}$ They offer support in a novel analysis of multivariate data from a uniquely large data-set for the 'two continua' model of mental health and well-being, ${ }^{2}$ as well as support for the hypothesis that psychological processes mediate the impact of biological, social and circumstantial factors on mental health. ${ }^{7}$ Although relatively few people experienced high

\begin{tabular}{|c|c|c|}
\hline & $\begin{array}{c}\text { Few life events } \\
n(\%)\end{array}$ & $\begin{array}{c}\text { Many life events } \\
n(\%)\end{array}$ \\
\hline \multicolumn{3}{|c|}{ All participants $(n=27397)$} \\
\hline \multicolumn{3}{|c|}{ Mental health problems } \\
\hline Low & 4898 (17.9) & $8546(31.2)$ \\
\hline High & 3252 (11.9) & 10701 (39.1) \\
\hline \multicolumn{3}{|c|}{ Low levels of rumination $(n=11834)$} \\
\hline \multicolumn{3}{|c|}{ Mental health problems } \\
\hline Low & $3548(30.0)$ & $5507(46.5)$ \\
\hline High & 755 (6.4) & 2024 (17.1) \\
\hline \multicolumn{3}{|c|}{ High levels of rumination $(n=15563)$} \\
\hline \multicolumn{3}{|c|}{ Mental health problems } \\
\hline Low & $1350(8.7)$ & 3039 (19.5) \\
\hline High & $2497(16.0)$ & $8677(55.8)$ \\
\hline
\end{tabular}

levels of well-being in the presence of mental health problems and, conversely, relatively few people with low levels of well-being were free from mental health problems, these two concepts were clearly not opposing poles on a single continuum, because they were associated with different causal processes. In one sense, this finding follows from the nature of the phenomena under examination; our measure of well-being incorporated physical, social and purely psychological well-being, whereas the assessment of anxiety and depression was more specifically psychological. It is perhaps therefore unsurprising that the two phenomena had different associations. However, although this makes the interpretation of the findings readily comprehensible, it does not reduce their potential significance.

These findings have practical as well as theoretical implications. They can (at least partially) help explain why people with good objective well-being, who are financially secure and in good relationships, can become depressed. These findings imply that 
Table 4 Contingent relationship between well-being and social activity, stratified by levels of adaptive coping, based on median splits of each variable

\begin{tabular}{|lcc|} 
& $\begin{array}{c}\text { Low level of } \\
\text { social activity } \\
n(\%)\end{array}$ & $\begin{array}{c}\text { High level of } \\
\text { social activity } \\
n(\%)\end{array}$ \\
$\begin{array}{l}\text { All participants }(n=27397) \\
\text { Well-being } \\
\text { Low } \\
\text { High }\end{array}$ & \\
\hline $\begin{array}{l}\text { Low level of adaptive coping }(n=10914) \\
\text { Well-being }\end{array}$ & $4995(32.8)$ & $4434(16.2)$ \\
Low & $4259(15.5)$ & $9709(35.4)$ \\
\hline High & $4875(44.7)$ & $1930(17.7)$ \\
\hline $\begin{array}{l}\text { High level of adaptive coping }(n=16483) \\
\text { Well-being } \\
\text { Low } \\
\text { High }\end{array}$ & $2713(24.9)$ \\
\hline
\end{tabular}

if bad things happen to them (they experience negative life events), and if they ruminate about those events, they are much more likely to become anxious or depressed. Clearly, this analysis can be extended to a wide range of other causes and mediators not included in this study. These findings can also partially explain how people who do not show signs of specific mental health problems such as anxiety and depression can have low levels of well-being or fail to flourish. If socially isolated and relatively lacking in adaptive coping responses (such as problem-solving and emotional self-regulation), people are more likely to experience low levels of well-being, but are unlikely to experience mental health problems if otherwise not exposed to the causal mechanisms described above.

\section{Implications of the study}

Practically, these findings suggest that interventions aimed at maximising well-being and interventions aimed at preventing or treating mental health problems should be complementary but different. We found that people who ruminated were three times more likely to develop anxiety or depression following negative life events, and people who lacked adaptive coping skills were three times more likely to have low subjective well-being in the face of social deprivation, but that these two pathways were independent of each other. These findings have important implications for current policies in health and social care. Specifically, they strongly suggest that conceptually distinct interventions are needed; services aimed at treatment and prevention of mental health problems may be very different from those needed to promote and improve subjective well-being. All such clinical decisions must be dictated by individual case formulations, but it seems reasonable to suggest that interventions and social policy aimed at improving social circumstances, addressing financial equity and social justice, building local communities, supporting families, and facilitating relationships on the societal level, and developing individuals' personal capacity to cope adaptively with stressful life circumstances may be best suited to developing subjective well-being. Local authority social services may have an immediate responsibility here, but genuine progress may necessitate wider societal and political action. Interventions aimed at preventing abuse, mitigating the consequences of traumatic personal life events, and evidence-based interventions targeting psychological processes such as rumination (including the now ubiquitous cognitive-behavioural therapy) may be best suited to helping people recover from mental health problems.
Peter Kinderman, PhD, Institute of Psychology, Health and Society, University of Liverpool; Sara Tai, ClinPsyD, School of Psychological Science, Manchester Eleanor Pontin, DClinPsych, Institute of Psychology, Health and Society, University of Liverpool; Matthias Schwannauer, PhD, School of Health in Social Science, Edinburgh; Ian Jarman, PhD, Paulo Lisboa, PhD, School of Computing and Mathematical Sciences, Liverpool John Moores University, Liverpool, UK

Correspondence: Professor Peter Kinderman, Institute of Psychology, Health and Society, University of Liverpool, Waterhouse Building, Liverpool L69 3GL, UK. Email: p.kinderman@liverpool.ac.uk

First received 24 Feb 2014, final revision 30 Sep 2014, accepted 6 Oct 2014

\section{Acknowledgements}

We thank the staff of BBC Lab UK for their support for the development and conduct of the study, and J. Rees for critical discussions and reading of the manuscript.

\section{References}

1 Keyes CLM. Mental health and/or mental illness? Investigating axioms of the complete state model of health. J Consult Clin Psychol 2005; 73: 539-48.

2 Westerhof GJ, Keyes CLM. Mental illness and mental health: the two continua model across the lifespan. J Adult Dev 2010; 17: 110-9.

3 Bacciu D, Etchells TA, Lisboa PJG, Whittaker J. Efficient identification of independence networks using mutual information. Comput Stat 2013; 28: 621-46.

4 Spirtes P, Glymour C, Scheines R. Causation, Prediction and Search (2nd edn). MIT Press, 2000.

5 Office for National Statistics. Labour Force Survey: Household Datasets, Spring Quarter. ONS, 2003.

6 Kinderman P. A psychological model of mental disorder. Harv Rev Psychiatry 2005; 13: 206-17.

7 Kinderman $\mathrm{P}$, Schwannauer M, Pontin E, Tai S. Psychological processes mediate the impact of familial risk, social circumstances and life events on mental health. PLOS One 2013; 8: e76564.

8 Milne BJ, Caspi A, Crump R, Poulton R, Rutter M, Sears MR, et al. The validity of the family history screen for assessing family history of mental disorders. Am J Med Genet B 2008; 50: 41-9.

9 Elliott R, Sahakian BJ, Herrod JJ, Robbins TW, Paykel ES. Abnormal response to negative feedback in unipolar depression: evidence for a diagnosis specific impairment. J Neurol Neurosurg Psychiatry 1977; 63: 74-82.

10 Murphy FC, Sahakian BJ, Rubinsztein JS, Michael A, Rogers RD, Robbins TW, et al. Emotional bias and inhibitory control processes in mania and depression. Psychol Med 1999; 29: 1307-21.

11 Chamberlain SR, Sahakian BJ. The neuropsychology of mood disorders. Curr Psychiatry Rep 2006; 8: 458-63.

12 National Development Team for Social Inclusion. Reviewing Measures of Social Exclusion. National Social Inclusion Programme, 2003.

13 Brugha TS, Cragg D. The list of threatening experiences: the reliability and validity of a brief life events questionnaire. Acta Psychiatr Scand 1990; 82 77-81.

14 Bernstein DP, Fink L. Initial reliability and validity of a new retrospective measure of child abuse and neglect. Am J Psychiatry 1994; 151: 1132-6.

15 Nolen-Hoeksema S, Wisco BE, Lyubomirsky S. Rethinking rumination. Perspec Psychol Sci 2008; 3: 400-24.

16 Kinderman P, Bentall RP. A new measure of causal locus: the internal, personal and situational attributions questionnaire. Pers Individ Dif 1996; 20: $261-4$.

17 Goldberg D, Bridges K, Duncan P, Grayson D. Detecting anxiety and depression in general medical settings. BMJ 1989; 297: 897-9.

18 Kinderman $\mathrm{P}$, Schwannauer M, Pontin E, Tai S. The development and validation of a general measure of well-being: the BBC well-being scale. Qual Life Res 2011; 20: 1035-42.

19 Pontin E, Schwannauer M, Tai S, Kinderman P. A UK validation of a general measure of subjective well-being: the modified BBC subjective well-being scale (BBC-SWB). Health Qual Life Outcomes 2013; 11: 150-9.

20 Goebel B, Dawy Z, Hagenauer J, Mueller JC. An approximation to the distribution of finite sample size mutual information estimates. IEEE Intl Conf comm South Korea 2005; 2; 1102-6.

21 Weich S, Brugha $T$, King M, McManus S, Bebbington $P$, Jenkins $R$, et al. Mental well-being and mental illness: findings from the Adult Psychiatric Morbidity Survey for England 2007. Br J Psychiatry 2011; 199: 23-8. 